\title{
Imaging in Acute Anterior Circulation Ischemic Stroke: Current and Future
}

\author{
Hyun Jeong Kim, MD, $\mathrm{PhD}^{1}$, Hong Gee Roh, $\mathrm{MD}, \mathrm{PhD}^{2}$ \\ ${ }^{1}$ Department of Radiology, Daejeon St. Mary's Hospital, College of Medicine, The Catholic University of Korea, Daejeon, Korea \\ ${ }^{2}$ Department of Radiology, Konkuk University Medical Center, Seoul, Korea
}

\begin{abstract}
Clinical trials on acute ischemic stroke have demonstrated the clinical effectiveness of revascularization treatments within an appropriate time window after stroke onset: intravenous thrombolysis (NINDS and ECASS-III) through the administration of tissue plasminogen activator within a 4.5-hour time window, endovascular thrombectomy (ESCAPE, REVASCAT, SWIFT-PRIME, MR CLEAN, EXTEND-IA) within a 6-hour time window, and extending the treatment time window up to 24 hours for endovascular thrombectomy (DAWN and DEFUSE 3). However, a substantial number of patients in these trials were ineligible for revascularization treatment, and treatments of some patients were considerably futile or sometimes dangerous in the clinical trials. Guidelines for the early management of patients with acute ischemic stroke have evolved to accept revascularization treatment as standard and include eligibility criteria for the treatment. Imaging has been crucial in selecting eligible patients for revascularization treatment in guidelines and clinical trials. Stroke specialists should know imaging criteria for revascularization treatment. Stroke imaging studies have demonstrated imaging roles in acute ischemic stroke management as follows: 1) exclusion of hemorrhage and stroke mimic disease, 2) assessment of salvageable brain, 3) localization of the site of vascular occlusion and thrombus, 4) estimation of collateral circulation, and 5) prediction of acute ischemic stroke expecting hemorrhagic transformation. Here, we review imaging methods and criteria to select eligible patients for revascularization treatment in acute anterior circulation stroke, focus on 2019 guidelines from the American Heart Association/American Stroke Association, and discuss the future direction of imaging-based patient selection to improve treatment effects.
\end{abstract}

Key Words: Acute ischemic stroke; Computed tomography, X-ray; Magnetic resonance imaging; Angiography; Perfusion; Collateral circulation

\section{INTRODUCTION}

Ischemic stroke is a major cause of death and disability worldwide. Ischemic stroke arises from compromised blood supply to the brain by occlusion of an artery due to hemodynamic factors or embolic causes, triggering cell death mechanisms evolving a series of complex spatial and temporal events occurring over minutes or even days. Within areas of severely reduced blood flow-the "core" of the ischemic territory-excitotoxic and necrotic cell death occurs within minutes, and tissue undergoes irreversible infarction in the absence of prompt reperfusion. However, cell death occurs relatively slowly in

\section{Correspondence to:} Hong Gee Roh, MD, PhD Department of Radiology, Konkuk University Medical Center, 120-1 Neungdong-ro, Gwangjin-gu, Seoul 05030, Korea

Tel: +82-2-2030-5496

Fax: +82-2-2030-5549

E-mail:hgroh@kuh.ac.kr

Received: October 20, 2021

Revised: December 25, 2021

Accepted: December 30, 2021
Copyright $\odot 2022$ Korean Society of Interventional Neuroradiology

This is an Open Access article distributed under the terms of the Creative Commons Attribution Non-Commercial License (http://creativecommons.org/licenses/by-nc/4.0) which permits unrestricted non-commercial use, distribution, and reproduction in any medium, provided the original work is properly cited.

pISSN 2093-9043 eISSN 2233-6273 
the peripheral zone surrounding the core supported by collateral circulation, termed the "penumbra", and cell viability is determined by the degree of ischemia, that is, the degree of collateral circulation and timing of reperfusion. Therefore, improving functional outcomes by preserving the salvageable brain with timely reperfusion is the treatment goal of acute ischemic stroke. Studies over 2 decades have demonstrated the therapeutic effect of revascularization treatments (intravenous thrombolysis [IVT] and endovascular thrombectomy [EVT]) in acute ischemic stroke and established guidelines to select eligible patients for revascularization treatments. ${ }^{1-8}$ Revascularization treatments are guided by the time from stroke onset, patient age, the severity of neurologic deficits, and neuroimaging findings, such as core, penumbra, and vessel status. ${ }^{8}$ Time is a key factor in applying revascularization treatment. IVT, through the administration of recombinant tissue plasminogen activator (rt-PA), is recommended by the guidelines within 4.5 hours of symptom onset and EVT within 6 hours with the presence of large vessel occlusion in neuroimaging. ${ }^{8}$ However, infarct progression is not entirely time-dependent. Infarction might be complete in less than 1 hour or may not be complete for hours or days depending on the collateral circulation, which shows regional and individual variance. ${ }^{9,10}$ Therefore, revascularization treatments can be futile or even dangerous when performed in the standard optimal time window but could be useful if performed in a later time window. ${ }^{11-13}$ Studies have shown that a large core combined with poor collaterals is a strong predictor of an unfavorable response to revascularization treatments, hemorrhagic complications, and poor functional outcome. ${ }^{14}$ Hence, exclusion of patients with a large core and poor collateral circulation could prevent futile and dangerous revascularization treatments. Conversely, good collateral circulation can limit core expansion and prolong the time the penumbra tissue at risk remains salvageable until revascularization treatment. Assessment of parenchyma (hemorrhage, core, and penumbra) and vessels (causative vessel and collateral circulation) by imaging became the cornerstone in the management of acute ischemic stroke. We review the imaging methods and criteria in the management of acute anterior circulation ischemic stroke that focuses on the 2019 guidelines from the American Heart Association (AHA)/ American Stroke Association (ASA) ${ }^{8}$ and discuss challenges and future directions in acute stroke imaging. The main eligibility recommendations for IVT and EVT in patients with acute ischemic stroke according to the 2019 guidelines from the AHA/ASA are summarized in Tables 1 and 2, respective$1 y_{1}^{8}$ and the imaging guidelines are summarized in Table $3 .^{8}$

\section{Assessment of Brain Parenchyma}

\section{Intracranial hemorrhage}

The first role of imaging is to diagnose ischemic stroke and exclude hemorrhagic stroke, which would contraindicate revascularization treatment. Computed tomography (CT) and magnetic resonance imaging (MRI) based on $\mathrm{T} 2^{*}$-weighted sequences are equivalent in detecting acute hemorrhage and a target thrombus in an occluded artery (susceptibility vessel sign). ${ }^{15-17}$ MRI has better accuracy for the detection of small petechial hemorrhagic transformation in infarction and previous hemorrhage and/or microbleeds. The high incidence (15-27\%) of cerebral microbleeds in patients receiving IVT has led to concern about the risk of symptomatic intracerebral hemorrhage in these patients after IVT. ${ }^{18}$ Studies have consistently reported that the risk of symptomatic intracerebral hemorrhage in patients with $>10$ cerebral microbleeds (30-47\%) is significantly greater than that in patients without cerebral microbleeds. ${ }^{18}$ A meta-analysis showed that the presence of cerebral microbleeds is associated with worse outcomes after IVT compared with patients without microbleeds. ${ }^{19}$ However, it is unclear whether these negative effects of cerebral microbleeds fully negate the benefit of IVT. Considering these studies, the 2019 guidelines newly recommend the following: IVT is reasonable in eligible patients who previously have had a small number (1-10) of cerebral microbleeds and may be reasonable in patients with $>10$ cerebral microbleeds if there is a potential for substantial benefit (Table 1).

\section{Core}

Assessment of the core on baseline neuroimaging is critical to select eligible patients for IVT and EVT (Tables 1, 2). Noncontrast CT (NCCT) is the most widely used first-line imaging tool for this purpose because of its advantages of quick acquisition and easy accessibility. NCCT findings of the core within a few hours after symptom onset are loss of gray-white matter differentiation because of a decreased CT density of gray matter structures. This appears on NCCT as a loss of distinction between the basal ganglia and adjacent white matter and as a blending of the densities of the cortex and underlying white matter in the insula (insular ribbon sign) and over the convexities (Fig. 1A, B). Sulcal effacement, 
another early NCCT finding, is produced by the subsequent swelling of the gyri. More advanced ischemia induces an obviously hypodense core on NCCT, which is considered irreversible tissue damage. Except for eligible patients for IVT within a 3-hour treatment window, patients with a core involving more than one-third of the middle cerebral artery (MCA) territory were excluded from revascularization treatment because the patients had a lower chance of good outcomes after revascularization treatment (Tables 1, 2). ${ }^{8}$ However, identification of patients following the $1 / 3$ rule

Table 1. Main eligibility recommendations for IVT in patients with acute ischemic stroke from the 2019 guidelines by the AHA/ASA ${ }^{8}$

\begin{tabular}{|c|c|}
\hline \multicolumn{2}{|l|}{ Indications } \\
\hline 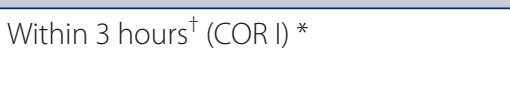 & $\begin{array}{l}\text { - Age } \geq 18 \text { years } \\
\text { - NIHSS score } \geq 6\end{array}$ \\
\hline 3 to 4.5 hours $^{\dagger}($ COR I)* & $\begin{array}{l}\text { - Age } \geq 18 \text { years and } \leq 80 \text { years } \\
\text { - Without a history of both diabetes mellitus and prior stroke } \\
\text { - NIHSS score } \geq 6 \text { and } \leq 25 \\
\text { - Not taking any OACs } \\
\text { - Without imaging evidence of ischemic injury involving more than one-third of the MCA } \\
\text { territory: IVT is recommended in the setting of early ischemic changes on NCCT of mild to } \\
\text { moderate extent (other than frank hypodensity) }\end{array}$ \\
\hline $\begin{array}{l}\text { Wake-up stroke or unclear time of onset } \\
>4.5 \text { hours from last known normal } \\
\text { state or at baseline state (COR Ila)* }\end{array}$ & $\begin{array}{l}\text { IVT can be beneficial when DWI lesion is smaller than one-third of the MCA territory and no } \\
\text { visible signal change on FLAIR }\end{array}$ \\
\hline CMB (COR Ila)* & $\begin{array}{l}\text { - Eligible patients who have previously had a small number (1-10) of CMBs demonstrated on } \\
\text { MRI, IVT is reasonable } \\
\text { - Eligible patients who have previously had a high burden of } \mathrm{CMBs}(>10) \text { demonstrated on } \\
\text { MRI, IVT may be associated with an increased risk of symptomatic ICH, and the benefits of } \\
\text { treatment are uncertain. IVT may be reasonable if there is the potential for substantial benefit }\end{array}$ \\
\hline
\end{tabular}

Contraindication

- NIHSS score 0-5 (COR III: no benefit)*

- NCCT: extensive regions of frank hypodensity (COR III: no benefit)*

- Acute ICH (COR III: harm)*

- Ischemic stroke within 3 months (COR III: harm)*

- Severe head trauma within 3 months (COR III: harm)*

- Posttraumatic infarction that occurs during the acute in-hospital phase (COR III: harm)*

- Intracranial/intraspinal surgery within 3 months (COR III: harm)*

- History of ICH (COR III: harm)*

- Symptoms and signs most consistent with a subarachnoid hemorrhage (COR III: harm)*

- Gastrointestinal malignancy (COR III: harm)*

- Gastrointestinal bleeding within 21 days (COR III: harm)*

- Coagulopathy: platelet count $<100,000 / \mathrm{mm}^{3}$, INR $>1.7$, aPTT $>40$ s, or PT >15 s (COR III: harm)*

- Infective endocarditis (COR III: harm)*

- Aortic arch dissection (COR III: harm)*

- Intra-axial intracranial neoplasm (COR III: harm)*

- Full treatment dose of LMWH within the previous 24 hours (COR III: harm)*

- Patients taking direct thrombin inhibitors or direct factor Xa inhibitors (COR III: harm)*

- Abciximab should not be administered concurrently with IV rt-PA (COR III: harm)*

- IV aspirin should not be administered within 90 minutes after the start of IV rt-PA (COR III: harm)*

IVT, intravenous thrombolysis; AHA, American Heart Association; ASA, American Stroke Association; COR, class of recommendation; NIHSS, National Institutes of Health Stroke Scale; OAC, oral anticoagulant; MCA, middle cerebral artery; NCCT, noncontrast computed tomography; DWI, diffusion-weighted magnetic resonance imaging; FLAIR, fluid-attenuated inversion recovery; CMB, cerebral microbleed; $\mathrm{MRI}$, magnetic resonance imaging; ICH, intracranial hemorrhage; INR, international normalized ratio; aPTT, activated partial prothrombin time; PT, prothrombin time; LMWH, low-molecular-weight heparin; IV, intravenous; rt-PA, recombinant tissue plasminogen activator. *COR amended to conform with American College of Cardiology/AHA 2015 Recommendation Classification System: COR I (strong), benefit>>>risk; COR Ila (moderate), benefit>>risk; COR IIb (weak), benefit $\geq$ risk; COR III (no benefit), benefit=risk; COR III (harm), risk> benefit. ${ }^{+}$When uncertain, the onset time should be considered the time when the patient was last known to be normal or at baseline neurological condition. 
was unreliable in clinical practice due to a considerable lack of interrater agreement in recognition and quantifying early ischemic NCCT findings. ${ }^{20}$ Addressing this limitation, a semiquantitative way to report early ischemic NCCT findings, the Alberta Stroke Program Early CT Score (ASPECTS), was devel- oped. ${ }^{21}$ ASPECTS evaluates the presence of early ischemic NCCT findings (loss of gray-white matter differentiation and sulcal effacement) in 10 regions of the MCA territory: 4 for subcortical structures (caudate, lentiform, internal capsule, insula) and 6 for cortical structures that are labeled M1-M3 at

Table 2. Main eligibility recommendations for EVT in patients with acute ischemic stroke from the 2019 guidelines by the AHA/ASA ${ }^{8}$

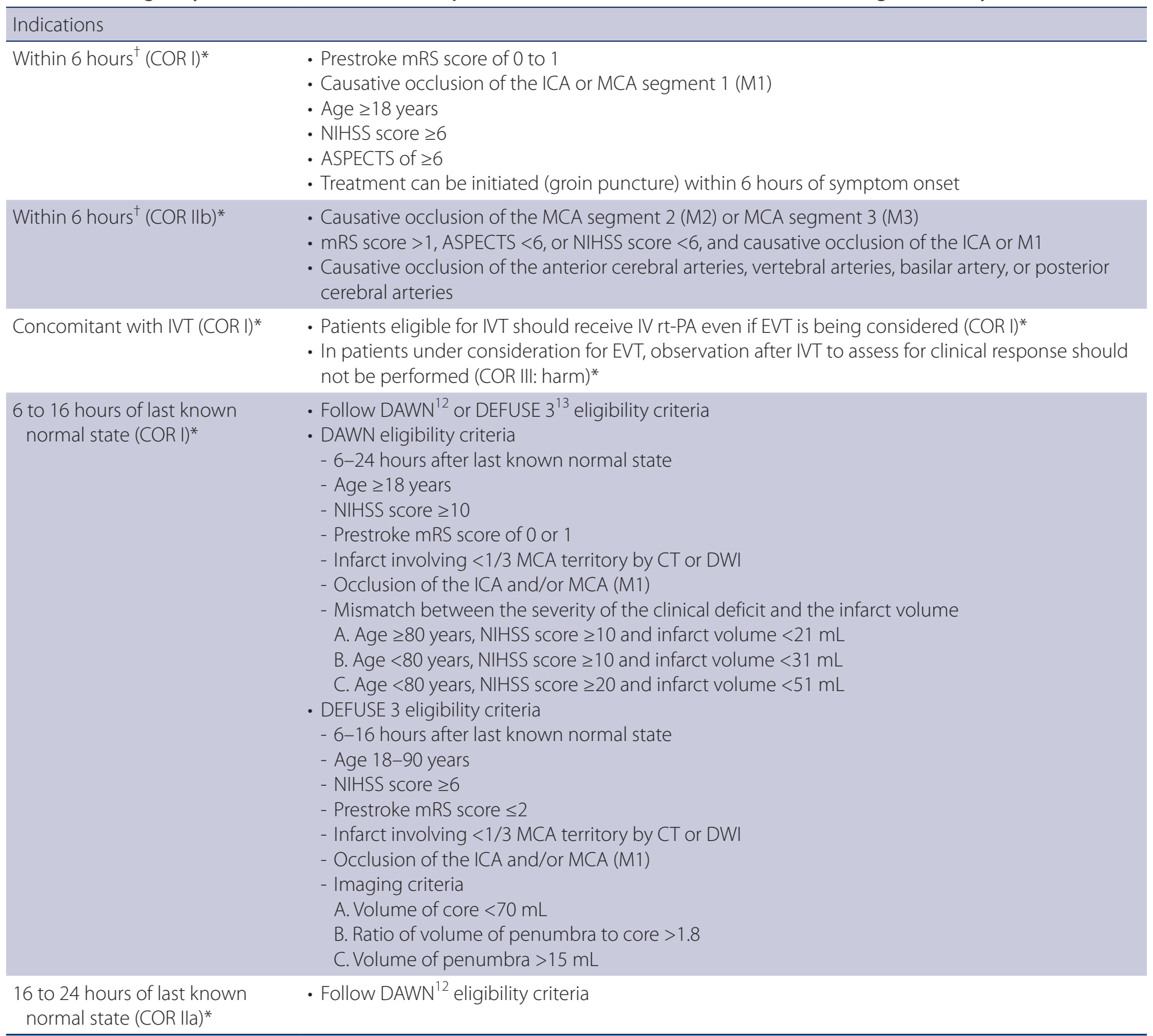

EVT, endovascular thrombectomy; AHA, American Heart Association; ASA, American Stroke Association; COR, class of recommendation; mRS, modified Rankin Scale; ICA, internal carotid artery; MCA, middle cerebral artery; NIHSS, National Institutes of Health Stroke Scale; ASPECTS, Alberta Stroke Program Early CT Score; CT, computed tomography; IVT, intravenous thrombolysis; IV, intravenous; rt-PA, recombinant tissue plasminogen activator; DWI, diffusion-weighted magnetic resonance imaging.

*COR amended to conform with American College of Cardiology/AHA 2015 Recommendation Classification System: COR I (strong), benefit>>>risk; COR Ila (moderate), benefit>>risk; COR IIb (weak), benefit zrisk; COR III (no benefit), benefit=risk; COR III (harm), risk>benefit. ${ }^{8}$ When uncertain, the time of onset time should be considered the time when the patient was last known to be normal or at baseline neurological condition. 


\section{Table 3. Imaging recommendations for IVT and EVT in patients with AIS from the 2019 guidelines by the AHA/ASA ${ }^{8}$}

Brain imaging
Initial imaging
1. All patients with suspected acute stroke should receive an emergency brain imaging
evaluation on first arrival to a hospital before initiating any specific therapy to treat AIS
2. Systems should be established so that brain imaging studies can be performed as quickly as
possible in patients who may be candidates for IVT or EVT or both
3. NCCT is effective to exclude ICH before IVT
4. MRI is effective to exclude ICH before IVT
5. CTA with CTP or MRA with DWI with or without MRP is recommended for certain patients

IVT eligibility

1. Administration of rt-PA in eligible patients without first obtaining MRI to exclude CMBs is recommended

2. In patients eligible for IVT, because the benefit of therapy is time-dependent, treatment should be initiated as quickly as possible and not delayed for additional multimodal neuroimaging, such as CTP and MRP

3. In patients with AIS who awoke with stroke symptoms or have unclear time of onset $>4.5$ hours from last known normal state or at baseline state, MRI to identify DWI-positive FLAIRnegative lesions can be useful for selecting those who can benefit from IVT within 4.5 hours of stroke symptom recognition

EVT eligibility-vessel imaging

1. For patients who otherwise meet criteria for EVT, noninvasive vessel imaging of the intracranial arteries is recommended during the initial imaging evaluation

2. For patients with suspected LVO who have not had noninvasive vessel imaging as part of their initial imaging assessment for stroke, noninvasive vessel imaging should then be obtained as quickly as possible (e.g., during rt-PA infusion if feasible)

3. In patients with suspected intracranial LVO and no history of renal impairment, who otherwise meet criteria for EVT, it is reasonable to proceed with CTA if indicated before obtaining a serum creatinine concentration

4. In patients who are potential candidates for EVT, imaging of the extracranial carotid and vertebral arteries, in addition to the intracranial circulation, may be reasonable to provide useful information on patient eligibility and endovascular procedural planning

5. It may be reasonable to incorporate collateral flow status into clinical decision-making in some candidates to determine eligibility for EVT

\section{EVT eligibility-multimodal imaging}

1. When selecting patients with AIS within 6 to 24 hours of last known normal status who have LVO in the anterior circulation, obtaining CTP or DWI, with or without MRP, is recommended to aid in patient selection for EVT, but only when patients meet other eligibility criteria from one of the RCTs that showed benefit from EVT in this extended time window

2. When evaluating patients with AIS within 6 hours of last known normal status with LVO and an ASPECTS of $\geq 6$, selection for EVT based on CT and CTA or MRI and MRA is recommended compared to performance of additional imaging such as CTP or MRP
$\mathrm{COR}^{*}$ New, revised, or unchanged

I Reworded for clarity from 2013 AIS guidelines $^{\dagger}$

I New recommendation

I Revised from 2013 guidelines ${ }^{\dagger}$

I Revised from 2013 guidelines ${ }^{\dagger}$

I New recommendation

I New recommendation

I New recommendation

Ila New recommendation

I Reworded for clarity from 2015 endovascular ${ }^{\ddagger}$

I Revised from 2015 endovascular ${ }^{\ddagger}$

lla New recommendation

Ila New recommendation

Ilb Revised from 2015 endovascular $^{\ddagger}$

I New recommendation

I New recommendation

IVT, intravenous thrombolysis; EVT, endovascular thrombectomy; AIS, acute ischemic stroke; AHA, American Heart Association; ASA, American Stroke Association; COR, class of recommendation; NCCT, noncontrast computed tomography; ICH, intracranial hemorrhage; CTA, computed tomography angiography; CTP, computed tomography perfusion imaging; MRA, magnetic resonance angiography; DWI, diffusionweighted magnetic resonance imaging; MRP, magnetic resonance perfusion imaging; rt-PA, recombinant tissue plasminogen activator; MRI, magnetic resonance imaging; CMB, cerebral microbleed; FLAIR, fluid attenuated inversion recovery; LVO, large vessel occlusion; RCT, randomized clinical trial; ASPECTS, Alberta Stroke Program Early CT Score; CT, computed tomography.

${ }^{*}$ COR amended to conform with American College of Cardiology/AHA 2015 Recommendation Classification System: COR I (strong), benefit>>>risk; COR Ila (moderate), benefit>>risk; COR IIb (weak), benefit $\geq$ risk; COR III (no benefit), benefit=risk; COR III (harm), risk>benefit. ${ }^{8}$ Data from the article of Jauch et al. (Stroke 2013;44:870-947). ${ }^{\ddagger}$ Data from the article of Powers et al. (Stroke 2015;46:30203035). 
the ganglionic level and M4-M6 at the supraganglionic level (Fig. 1C). The rater subtracts 1 point for every abnormal region, and the total score ranges from 10 (without early ischemic change) to 0 (core involving the whole MCA territory). A recent meta-analysis performed a central reading of all baseline NCCT from the major 5 EVT trials in $2015^{3-7}$ and found a clear benefit of EVT in patients with ASPECTS $\geq 6,{ }^{22}$ which has been adopted in current guidelines. However, for accuracy, the ASPECTS has the innate problem that NCCT itself has a significantly lower sensitivity than MR to depict cores in early time periods, with an overall sensitivity of $57-71 \%$ in the first 24 hours of symptom onset and only 12\% in the first 3 hours. The 10 regions in the MCA territory defined in the ASPECTS are based on anatomical structures, and thus, the individual regions cover different amounts of brain tissue. Therefore, the correlation of the ASPECTS with the core volume of diffusion-weighted MR imaging (DWI) varies considerably depending on the lesion location. As core volume is a strong predictor of functional outcome, the regional unequal weighing of the ASPECTS could compromise treatment decisions. Contrary to the low sensitivity of NCCT to depict cores, DWI has 73-92\% sensitivity to depict cores in the first 3 hours and up to $95-100 \%$ sensitivity in the first 6 hours after ischemic onset (Figs. 1A, 1B, 1J, 1K, 2A, 3A, 3B, 4A). 15,16 Cytotoxic edema (condition of restricted diffusion) in the ischemic brain occurs when cerebral blood flow (CBF) decreases under 30/100 g/min increased DWI signal intensity within minutes after ischemic onset. The DWI signal change is more conspicuous than NCCT, so direct measurement of early core volume is possible (Fig. 1). Current guidelines do not present DWI criteria for the recommendation of IVT or EVT in patients within 6 hours after symptom onset. Studies and clinical trials have shown that a DWI-ASPECTS $\geq 5$ and DWI lesion volume $<70 \mathrm{~mL}$ could be optimal thresholds to predict the benefit of EVT., Manual outlining of DWI lesions has been used to extract volume data, but this method is time-consuming and operator dependent. To overcome this limitation, automated estimation of the core using an apparent diffusion coefficient threshold of $620 \times 10^{-6} \mathrm{~mm}^{2} /$ second is currently applied in practice. ${ }^{23}$

\section{Penumbra and mismatch concepts}

Considering hemorrhagic complications and the considerable rate of futile treatment, ${ }^{3-7}$ the ideal patient for revascularization treatment should have a salvageable brain (penumbra), which is a brain at risk of infarction due to critical hypoperfusion that can survive with reperfusion. Perfusion imaging using $\mathrm{CT}$ or MRI techniques is the most used method for the assessment of penumbra. CT perfusion (CTP) and MR perfusion (MRP) obtained by continuous scanning over 45 to 90 seconds after administration of contrast material provide information on tissue enhancement according to time, which represents the amount of contrast material, that is, the amount of blood reaching the brain. Cerebral blood volume (CBV), CBF, time-to-maximum (Tmax), and mean transit time (MTT) are displayed by calculation based on tissue density (or signal intensity) time curves of perfusion imaging. Studies have tried to identify critically hypoperfused tissue and core by applying thresholds for CBV, CBF, MTT, or Tmax. The mismatch area between the critically hypoperfused tissue and core is considered the penumbra, which is called the 'target mismatch' because the mismatch area is a target for revascularization treatment. A Tmax threshold $>6$ seconds ( $\operatorname{Tmax}>6 \mathrm{~s}$ ) has been a reasonable estimate of critically hypoperfused tissue in the absence of reperfusion (Figs. 1H, 3D, 4C). ${ }^{24}$ The mismatch area can be estimated with the difference between the volume of MRP Tmax $>6 \mathrm{~s}$ and the DWI lesion when we use MRI as a baseline imaging tool. However, estimating the penumbra when the baseline imaging tool is $\mathrm{CT}$ is not simple because we cannot measure the volume of the core on CT images due to the inconspicuousness of early NCCT findings. Studies comparing CTP maps with DWI lesions showed that a threshold of $<30 \%$ of the contralateral mean CBF (CBF <30\%) was a reasonable CTP threshold of the core. ${ }^{25}$ Therefore, in the CT workup, the mismatch area can be estimated with a difference between the volume of Tmax $>6 \mathrm{~s}$ and $C B F<30 \%$ (Figs. 1A, 1B, 1H, 1J, 1K, 2A, 2C-F, 3A, 3B, 3D, 3E, 4A, 4C, 4D). Clinical trials using a target mismatch (EPITHET, ${ }^{26}$ SWIFT-PRIME, ${ }_{1}^{5}$ EXTEND-IA, etc.) to select patients for revascularization treatments showed a greater treatment effect relative to clinical trials that did not use the target mismatch concept (NINDS, ${ }^{1}$ ESCAPE, ${ }^{3}$ REVASCAT, ${ }^{4}$ MR CLEAN, ${ }^{6}$ etc.). In 2018, DAWN ${ }^{12}$ and DEFUSE $3^{13}$ trials showed the benefit of EVT compared with medical treatment in patients last known to be normal 6 to 24 hours (DAWN) and 6 to 16 hours (DEFUSE 3) earlier. The DAWN trial used CTP to estimate the core with a threshold of CBF $<30 \%$ and enrolled patients with a small core infarction that was mismatched to the clinical severity (NIHSS). DEFUSE 3 enrolled patients with a target mismatch on CTP or MRP. The 2019 guidelines promptly accepted the inclusion criteria of DAWN and DEFUSE 3 to select eligible patients for EVT in 

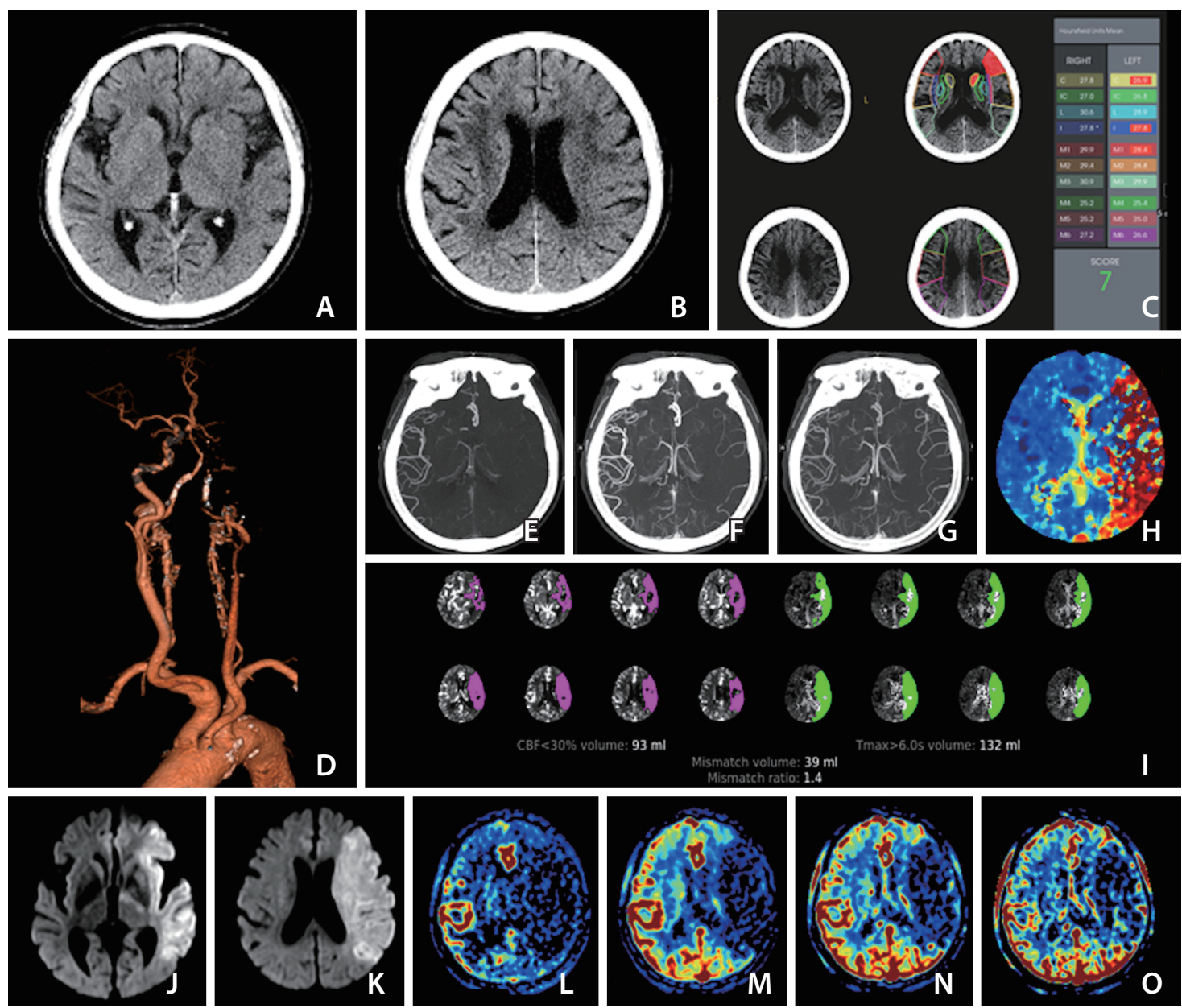

Fig. 1. Images at admission of an elderly patient who presented with dysarthria and right-sided weakness at 2 and half hours after last known normal state. The premorbid modified Rankin scale score of this patient was 0, and the National Institutes of Health Stroke Scale score at admission was 23. (A) Ganglionic level and (B) supraganglionic level, noncontrast computer tomography (NCCT) images show loss of gray-white matter differentiation at insula, M2, M3, M4, and M5 of the left middle cerebral artery territory, and the Alberta Stroke Program Early CT Score (ASPECTS) is 5 visually. (C) Computer-aid automated ASPECTS is 7 (RAPID, RapidAI ${ }^{\circledR}$, Menlo Park, CA, USA). (D) Computed tomography angiography (CTA) shows occlusion of the left internal carotid and middle cerebral arteries. $(\mathbf{E}-\mathbf{G})$ The first $(\mathbf{E})$, second $(\mathbf{F})$, and third phase $(\mathbf{G})$ collateral images derived from multiphase CTA show a filling delay of 2 phases in the affected hemisphere with a significantly reduced number of vessels in the ischemic territory (multiphase CTA collateral score 2). (H) CT perfusion (CTP) Tmax threshold $>6$ seconds (Tmax $>6$ s) map indicates near whole left middle cerebral artery territory as penumbra (red zone). (I) Automated software (RAPID, RapidA ${ }^{\circledR}$ ) demonstrates the volume data of core and penumbra and mismatch ratio between the penumbra and core, which are $93 \mathrm{~mL}$ with a threshold of CTP cerebral blood flow $<30 \%$ and $132 \mathrm{~mL}$ with threshold of a Tmax > $6 \mathrm{~s}$ and 1.4, respectively. (J) Ganglionic level and (K) supraganglionic level, diffusion-weighted magnetic resonance images (DWI) obtained 10 minutes after CT scan show a large core involving the entire left middle cerebral artery territory. (L-O) Images of arterial (L), capillary (M), early venous $(\mathbf{N})$, and late venous $(\mathbf{O})$ phases of the collateral map derived from dynamic contrast-enhanced magnetic resonance angiography show very poor collateral perfusion status (MAC score of 0$)^{38}$ defined as collateral perfusion delay/defect more than one-half of affected middle cerebral artery territory remained until the late venous phase. DWI $(\mathbf{J}, \mathbf{K})$ and the collateral map $(\mathbf{L}-\mathbf{O})$ indicate that the core rapidly progresses to infarction. According to current guidelines, she should receive intravenous thrombolysis (IVT) after scanning NCCT (A, B). Even though the visually estimated NCCT ASPECTS is 5, it is not easy to exclude her from endovascular thrombectomy (EVT) because it is difficult to accept the NCCT ASPECTS. In addition, the automated ASPECTS is $7(\mathbf{C})$, which is an eligible score for EVT. The result of automated software is dependent on the quality of the learning material, which is used as ground truth in deep learning. Automated ASPECTS is limited in accuracy due to the inconspicuousness of NCCT. We can see another result of the automated core size estimated with a threshold of CTP cerebral blood flow $<30 \%$ (I), which is different from the results of visual and automated estimation. It is inevitably limited to measuring cores containing heterogeneous factors regarding occluded duration, collateral status, and cellular composition with different ischemic resistance as a fixed threshold value. This indirect method of core assessment is also due to the inconspicuousness of NCCT, and this is a major limitation of CT workup in acute ischemic stroke despite its availability and rapidity. However, DWI shows a far-progressed large infarction directly and clearly $(\mathbf{J}, \mathbf{K})$. We do not need to hesitate excluding her from EVT. At current guidelines, there is no basis for excluding her from IVT or for further imaging work-up except NCCT. We have no choice but to inject recombinant tissue plasminogen activator (rt-PA). However, through this case, we can understand the significant ratio of futile or dangerous treatments in current guidelines. 
the late time window (Table 2).

Based on the WAKE-UP trial in 2017,27 the 2019 guideline included another imaging-based concept called DWI-fluid-attenuated inversion recovery (FLAIR) mismatch, which is a DWI-positive and FLAIR-negative lesion, for identification eligibility for IVT in patients who awoke with stroke or have an unclear onset time $>4.5$ hours from the last known well or at the baseline state (Tables 1, 3). FLAIR hyperintensity, which requires an increase in total tissue water (vasogenic edema), lags behind DWI hyperintensity due to diffusion restriction
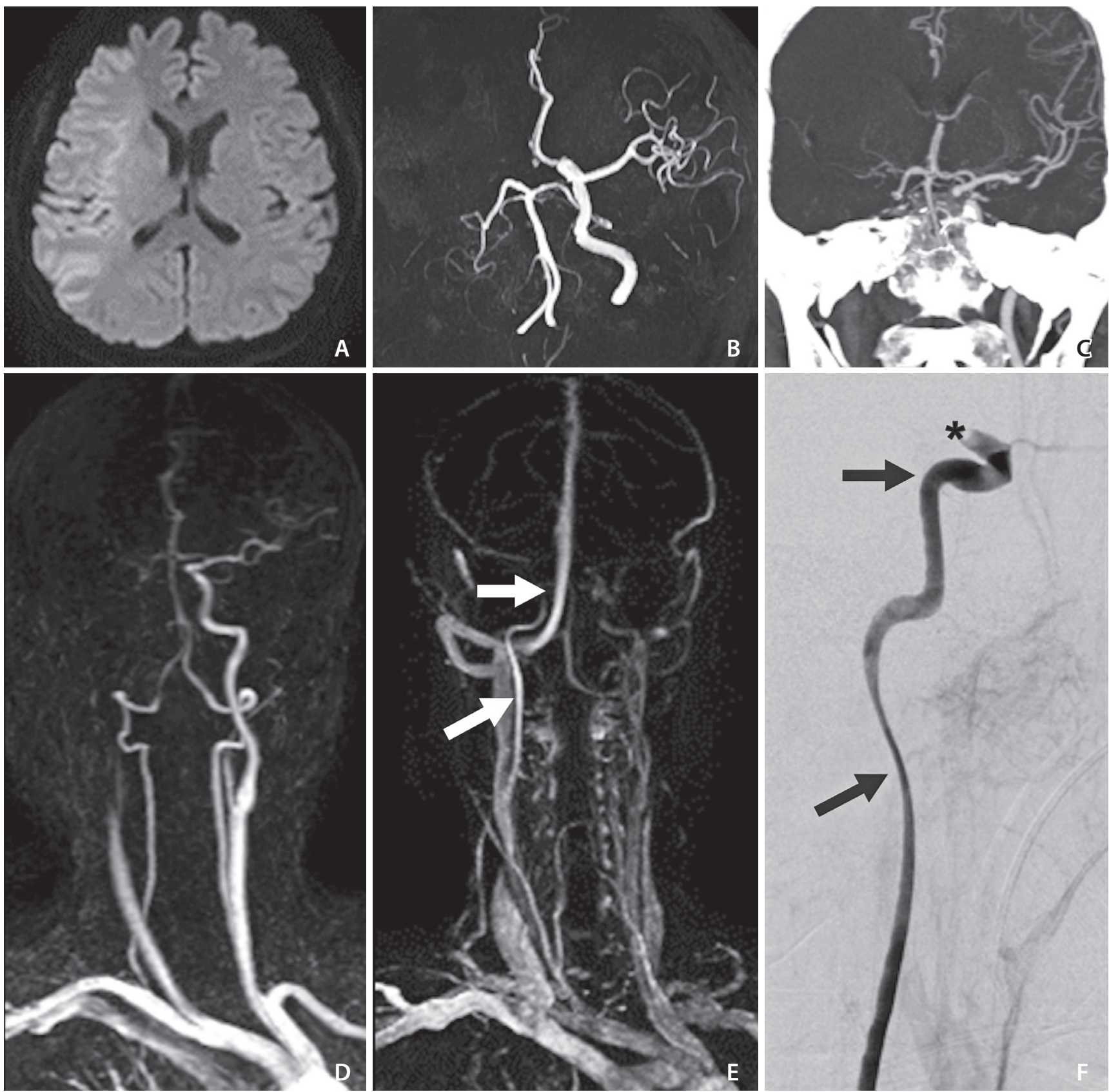

Fig. 2. Images of an elderly patient who developed left-sided weakness 2 hours prior. (A) Diffusion-weighted magnetic resonance imaging at admission shows a core involving the whole right middle cerebral artery territory. (B) Time-of-flight magnetic resonance angiography (TOF-MRA) and (C) computed tomography angiography (CTA) show occlusion from the right proximal internal carotid artery (ICA) to the ipsilateral middle cerebral artery. (D) Early phase image of dynamic contrast-enhanced magnetic resonance angiography (DCE-MRA) shows the same finding as that of TOFMRA and CTA. (E) More delayed phase image than (D) of DCE-MRA reveals delayed flow of the ICA reaching the cavernous segment (white arrows). (F) Catheter digital subtraction angiography reveals the patent right proximal ICA (black arrows) and occlusion of the C1 segment of the right ICA (*). 
(cytotoxic edema) as the stroke evolves. DWI hyperintense lesions without corresponding FLAIR hyperintensity indicated a stroke within 4.5 hours of onset with an $83-87 \%$ positive predictive value, ${ }^{28}$ and the WAKE-UP trial could use the DWIFLAIR mismatch concept to select eligible patients for IVT in the late time window. Recently, Broocks et al. ${ }^{29}$ showed that quantitative net water uptake between ischemic core and mirrored contralateral brain, which was calculated based on CT density, was comparable to DWI-FLAIR mismatch for identification eligibility for IVT in this patient group.

\section{Assessment of Vessels}

\section{Causative vessels and target thrombus}

Current guidelines adhere to immediate administration of intravenous rt-PA after core evaluation (ASPECTS or core volume) if the patient meets the inclusion criteria within 4.5 hours after symptom onset and has no contraindications (Table 1). Delay of IVT for additional imaging workup is not permitted, so the next imaging work-up proceeds during rt-PA administration. The next goal of imaging is to find the occluded vessel that is the cause of the infarction to select eligible patients for EVT. Contrast-enhanced CT angiography (CTA) and MR angiography (MRA) are widely used for this purpose and accomplish the task with less than 90 seconds of acquisition time. Contrast-enhanced CTA (Figs. 1D, 2C) and MRA (Figs. 3C, 4B) can directly show not only the causative vessel but also the overall information of cervicocerebral arteries, such as anatomy of the aortic arch, tortuosity of the proximal extracranial neck vessels, atherosclerosis of the carotid bifurcation, cervicocephalic dissection, or other cerebrovascular diseases, such as vasculitis, intracranial aneurysm,
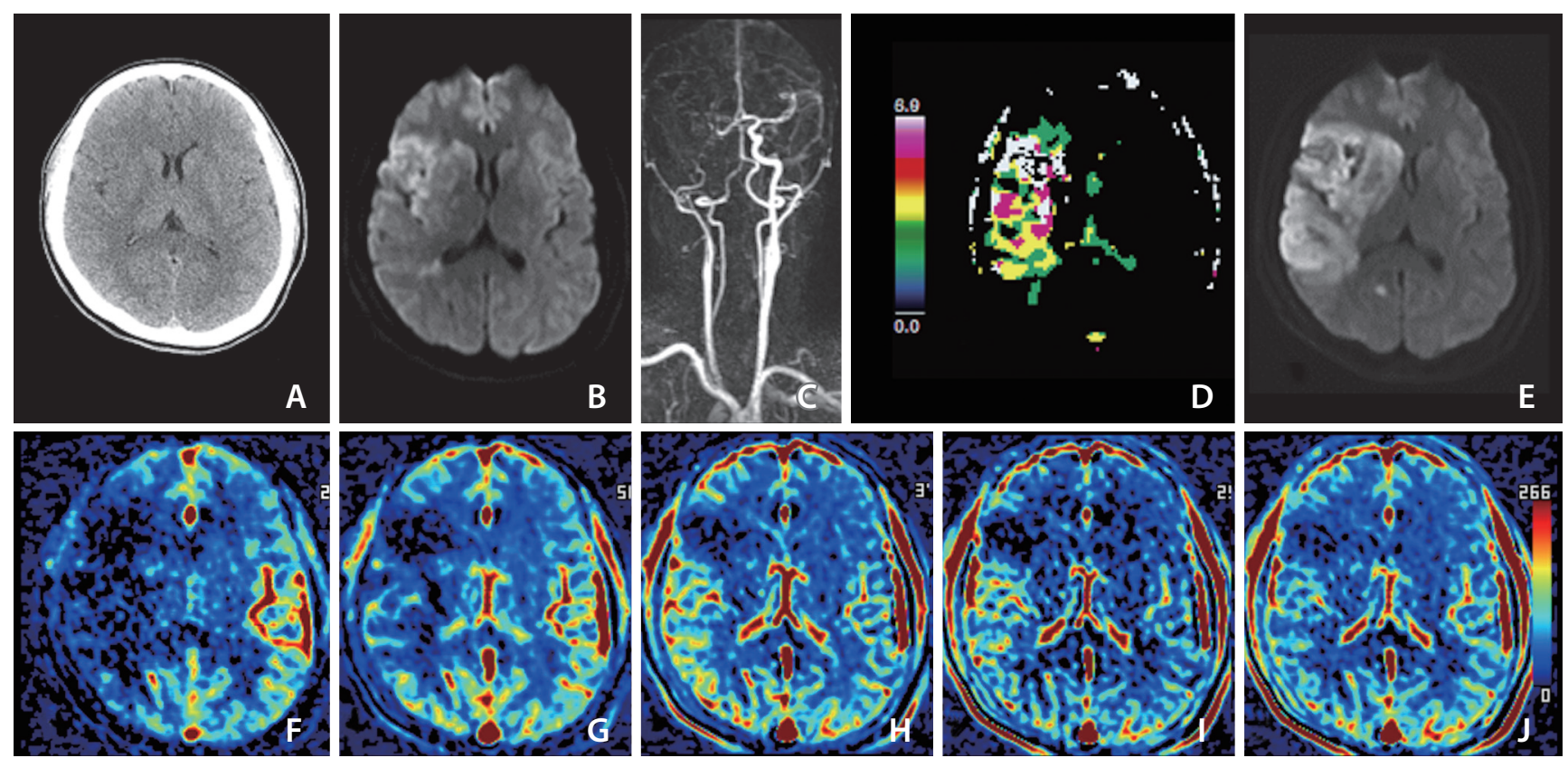

Fig. 3. Images of a middle aged patient who developed left-sided weakness 30 minutes prior. Her premorbid modified Rankin scale score was 0 , and the National Institutes of Health Stroke Scale score at admission was 11. She underwent intravenous thrombolysis followed by intraarterial thrombectomy, but recanalization of the occluded arteries was not achieved, as shown by a modified Thrombolysis in Cerebral Infarction scale score of 2a. (A) Noncontrast computed tomography image at admission shows less distinction of the right basal ganglia than the left basal ganglia. (B) Diffusion-weighted magnetic resonance imaging (DWI) at admission shows a core involving the insula, caudate nucleus, basal ganglia, internal capsule, M1, cortical area of $\mathrm{M} 2$ and $\mathrm{M} 3$, and some periventricular white matter of M3 on the right middle cerebral artery territory. (C) Dynamic contrast-enhanced magnetic resonance angiography (DCE-MRA) at admission shows occlusion of the right internal carotid and middle cerebral arteries. (D) MR perfusion Tmax threshold $>6$ seconds (Tmax $>6$ s) map at admission shows penumbra (white zone) matched with the core. (E) DWI on Day 1 shows a significantly increased extent of the core on DWI at admission due to reperfusion failure. $(\mathbf{F}-\mathbf{J})$ Images of arterial $(\mathbf{F})$, capillary $(\mathbf{G})$, early venous $(\mathbf{H})$, late venous (I), and delay $(\mathbf{J})$ phases of collateral map derived from DCE-MRA show intermediate to poor collateral perfusion status (MAC score of 2$)^{38}$ defined as collateral perfusion delay/defect more than one-half of affected middle cerebral artery territory in the capillary phase and equal to or less than one-half in the early venous phase. Because there was no target mismatch on Tmax $>6 s(\mathbf{D})$, despite no penumbra on Tmax $>6 s$, the extent of the baseline core increased as much as the hypoperfused area on the capillary phase of the collateral map at admission, and the extent of the baseline core coincided with the hypoperfused area in the early venous phase. 
or arteriovenous malformation. These factors affect EVT decisions and are important to neurointerventionists when planning an endovascular procedure. Modern EVT practice uses multiple devices and techniques tailored to the needs of a specific situation. Previous information on the aortic arch, neck vessels, and intracranial circulation, including thrombus location, helps in choosing appropriate devices and techniques, enabling more rapid and safe recanalization. Timeof-flight (TOF) MRA is a vascular imaging method that does not use contrast material (Fig. 2B). The sensitivity and specificity for detecting arterial occlusion are lower than those of contrast-enhanced MRA, and the acquisition time is relatively lengthy (approximately 3-4 minutes). However, it can be a good imaging option to detect the causative vessels in patients with renal impairment. Contrast-enhanced CTA and MRA and TOF-MRA show only a single static vascular status restricted to the early arterial phase. Slow-flow dynamics due to distal steno-occlusive lesions can result in pseudoocclusion appearing upstream of the exact steno-occlusive lesion on these single-phase angiographies (Fig. 2B, C). Multiphase CTA and dynamic contrast-enhanced (DCE)-MRA provide dynamic flow information to overcome this limitation and identify the location of the steno-occlusive lesion, which is misled on arterial phase images, on delayed phase images more exactly (Fig. 2D, E). ${ }^{30}$

A thrombus lodged in the occluded artery is shown as a hyperdense artery on NCCT, which is called a hyperdense artery sign due to increase density of thrombus by clotted blood. The hyperdense artery sign is present in $<50 \%$ of patients on standard 3-5 mm-slice NCCT but is highly specific for vessel occlusion with a specificity of $90-100 \% .{ }^{31}$ This finding is shown as a susceptibility vessel sign on SWI, which is defined as a hypointense signal that exceeds the diameter of the contralateral artery located at the site of the thrombus. $\mathrm{CT}$ hyperdensity and paramagnetic susceptibility of thrombi are due to the higher proportion of RBC-rich clots that have more concentrated deoxyhemoglobin than fibrin-rich clots. Although it is still debatable, this fact represents the association of the hyperdense artery sign (or susceptibility vessel sign) with the cardioembolic cause of acute ischemic stroke. ${ }^{31}$ The role of identifying vessel occlusion of the hyperdense artery sign on NCCT is not as significant today as it was when CTA was not popular. However, direct visualization of the location and extent of the thrombus and assuming the cause of stroke are quite useful information for neurointerventionists to decide on the appropriate thrombectomy devices, position the devices, and plan the subsequent procedure.

\section{Collateral circulation}

Cerebral collaterals are alternative vascular channels for maintaining blood perfusion to the ischemic brain distal to arterial occlusion. In cerebral ischemia due to large-vessel occlusion, cell viability depends on the collateral circulation, which has high interindividual variability. Therefore, collateral estimation provides the most potentially powerful prognostic insights. Estimation of the core and penumbra by NCCT, DWI, and perfusion imaging is an indirect method of collateral estimation. Initially, contrast-enhanced CTA was studied for direct collateral estimation. The arterial scoring method based on this single-phase CTA had a critical limitation of underestimating the collaterals with a longer transit time due to early triggering of a static acquisition. ${ }^{32}$ Patients excluded from revascularization treatment because of poor collaterals on single-phase CTA may have quite good collaterals that simply arrived after CTA acquisition. Multiphase CTA overcame this limitation by 3 consecutive acquisitions after administration of contrast material to capture the delayed flow (Fig. 1E-G). ${ }^{33}$ A secondary analysis from the MR CLEAN ${ }^{6}$ trial showed that a significant benefit of EVT was found in patients with good and intermediate collaterals on single-phase CTA and none in patients with poor collaterals. ${ }^{34}$ The ESCAPE trial used multiphase CTA in imaging selection of eligible patients for EVT and showed that it helped to determine the clinical outcome. ${ }^{3}$ Based on these studies, the AHA/ASA recommends that the incorporation of collateral flow status into clinical decision-making in some candidates to determine eligibility for EVT may be reasonable in the 2018 guidelines, and they kept this recommendation in 2019. ${ }^{8}$ However, the benefit of collateral-based patient selection over the perfusion imaging-based method in selecting patients who are most likely to benefit from EVT is questionable. The treatment effect of EVT in the ESCAPE trial ${ }^{3}$ was inferior to the SWIFT-PRIME ${ }^{5}$ and EXTEND-IA $A^{7}$ trials, which used perfusion imaging to select patients with a target mismatch. A post hoc analysis of the SWIFT-PRIME ${ }^{5}$ trial found a benefit for patients undergoing EVT regardless of collateral status on single-phase CTA. ${ }^{35}$ We suppose that the inferiority of the collateral-based method compared to the perfusion imaging-based method was due to the limitations of current collateral imaging methods. Neither single-phase CTA nor multiphase CTA provides parenchymal perfusion information but only pial arterial infor- 
mation at 3 fixed time points according to $C T$ table speed, which does not reflect individual hemodynamics, and it is still unclear whether the flow reaches and permeates brain tissue (Fig. 1E-G). The venous outflow concept has emerged from this limitation of artery-oriented collateral estimation. Brain perfusion is not only determined by the arterial inflow but also by downstream resistance due to tissue pressure or capillary and venous characteristics. Faizy et al. ${ }^{36}$ showed that favorable venous outflow profiles, which were assessed by counting veins on CTA, correlate with favorable tissue-level collaterals and clinical outcomes. However, venous outflow profiles based on CTA have the similar limitation of underestimating the collaterals with a longer transit time or slow hemodynamics like single-phase CTA. A novel multiphase collateral imaging method, called collateral map, using dynamic signals of dynamic susceptibility contrast-enhanced MRP (DSC-MRP) and DCE-MRA was developed recently. ${ }^{37}$ The collateral map consists of images of arterial, capillary, early venous, late venous, and delay phases determined according to the hemodynamics of each patient and provides more detailed information on brain perfusion and vessels from arterial phase to venous phase (Figs. 1L-O, 3F-J, 4E-I). Kim et al. ${ }^{38}$ showed the clinical prognostic value of the collateral map in acute ischemic stroke due to anterior circulation large vessel occlusion. We expect that sophisticated collateral perfusion imaging with temporal information can provide precise information on the penumbra (Fig. 3). If this approach is clinically validated, then it will be possible to estimate the causative vessel, penumbra, and collateral perfusion status of a patient based on 1 imaging acquisition (DCE-MRA). And the MRI protocol can be simplified with a $<4$ minutes acquisition time composed of DWI (acquisition time, 34 seconds), SWI (97 seconds), and DCE-MRA (60-90 seconds). Additionally, Lee et al. ${ }^{39}$ showed the predictability of symptomatic hemorrhagic transformation (parenchymatous hematoma 2) with collateral map and permeability imaging derived from DCE-MRA in patients with poor collateral perfusion status.

The prominent vessel sign, which is demonstrated as prominent cortical and/or medullary veins in ischemic brains on SWI, has been introduced as an imaging marker for collateral assessment. ${ }^{31,40}$ The prominent vessel sign has been positively correlated with the amount of deoxyhemoglobin in cerebral veins secondary to an increased oxygen extraction fraction of the ischemic brain, which shortens $\mathrm{T}^{*}$ relaxation and decreases the signal intensity of the veins in the ischemic region with respect to that in a normal brain. ${ }^{31}$ Therefore, ischemic tissue with poor collaterals requires more oxygen and contains more deoxyhemoglobins in tissue vessels, demonstrating a more prominent vessel sign on SWI than that of tissue with good collaterals. The prominent vessel sign is another indirect finding of collateral perfusion status, such as various perfusion parameters. Lu et al. ${ }^{41}$ reported that mismatch between SWI (quantitatively measured volume of a region with a prominent vessel sign) and DWI showed good consistency with target mismatch of perfusion imaging and provided a reliable method to reflect the penumbra. SWI is an essential sequence in the baseline MRI protocol used for evaluating patients with acute ischemic stroke to exclude patients with intracranial hemorrhage from revascularization treatment. Therefore, assessment of collaterals and penumbra by SWI is an efficient triage option in time-pressured environments of acute ischemic stroke care, particularly in patients for whom contrast material is not recommended.

\section{CHALLENGES AND FUTURE}

\section{Tissue Viability}

The major challenge of current imaging evaluations in acute stroke is the accuracy in estimating tissue viability. EVT is currently recommended in patients with small cores, which is estimated with imaging thresholds of NCCT ASPECTS $\geq 6$, DWI ASPECTS $\geq 5$, or DWI lesion or PWI lesion (CBF <30\%) volume $<70 \mathrm{~mL}$. The underlying assumption is that patients with a large core have less salvageable tissue and are thus unlikely to benefit and more likely to suffer hemorrhagic transformation from revascularization treatment, as most core tissue is considered irreversibly damaged. In current guidelines, patients with cores involving more than one-third of the MCA territory, which is identified by imaging thresholds of NCCT ASPECTS $<7$, DWI ASPECTS $<6$, or DWI lesion or perfusion lesion (Tmax $>8-10$ seconds) volume $>70-100 \mathrm{~mL}$, are not included in EVT. The progression of ischemic tissue is highly complex and individual. Jones et al showed that CBF thresholds for irreversible tissue damage depend on the duration of occlusion, ${ }^{42}$ and Marcoux et al. ${ }^{43}$ showed that tissue tolerance to ischemia differs between tissue types. Applying fixed thresholds across all time points from stroke onset is not reliable; therefore, studies have shown that CTP thresholds are not accurate for estimating the core ${ }^{44}$ or penumbra ${ }^{45}$ and can lead to erroneous treatment decisions. The 
potential for DWI lesion reversibility with rapid reperfusion, especially in less than 3 hours after stroke onset, shows also the limitation of core estimation by DWI, ${ }^{46}$ which indicates that excluded patients from revascularization treatments due to large DWI lesions may be eligible for treatment (Fig. 4). DWI hyperintensity does not always represent cell death but tissue with cytotoxic edema induced by severe ischemia. There are numerous and various cells with heterogeneous fates in the DWI lesions that can evolve to full infarction, partial infarction, or normal tissue outcome depending on the speed and quality of reperfusion in DWI lesions. Follow-up infarct volume, which is conceptualized as target mismatch in the present stroke imaging evaluation, is a strong independent predictor of functional outcome. ${ }^{47}$ Currently, we do not have a tool for predicting the follow-up infarct volume except perfusion thresholds for penumbra estimation, such as
Tmax $>6$ seconds, which we already reviewed as a limitation. Even if there are limitations, only imaging can distinguish regions of the brain likely to be irreversibly injured versus potentially salvageable, which are essential elements of the guidelines for precise treatment. Infarct growth is more dependent on collateral status than the elapsed time: infarction progresses slowly in good collaterals (slow progressor) (Fig. 4) and is accelerated in poor collaterals (fast progressor) (Fig. 1). The baseline cores, even with the same time point and same volume, have different fates according to collateral status. Dr. Albers noted that a factor for better results in late window trials (DAWN ${ }^{12}$ and DEFUSE $3^{13}$ ) compared to early window trials (ESCAPE, ${ }^{3}$ REVASCAT, ${ }^{4}$ SWIFT-PRIME, ${ }^{5}$ MR CLEAN, ${ }^{6}$ EXTEND-I $A^{7}$ ) in treatment effect of EVT was that a substantial percentage (about 50\%) of patients have very slow growth of the ischemic core for up to 12 hours or longer due to the
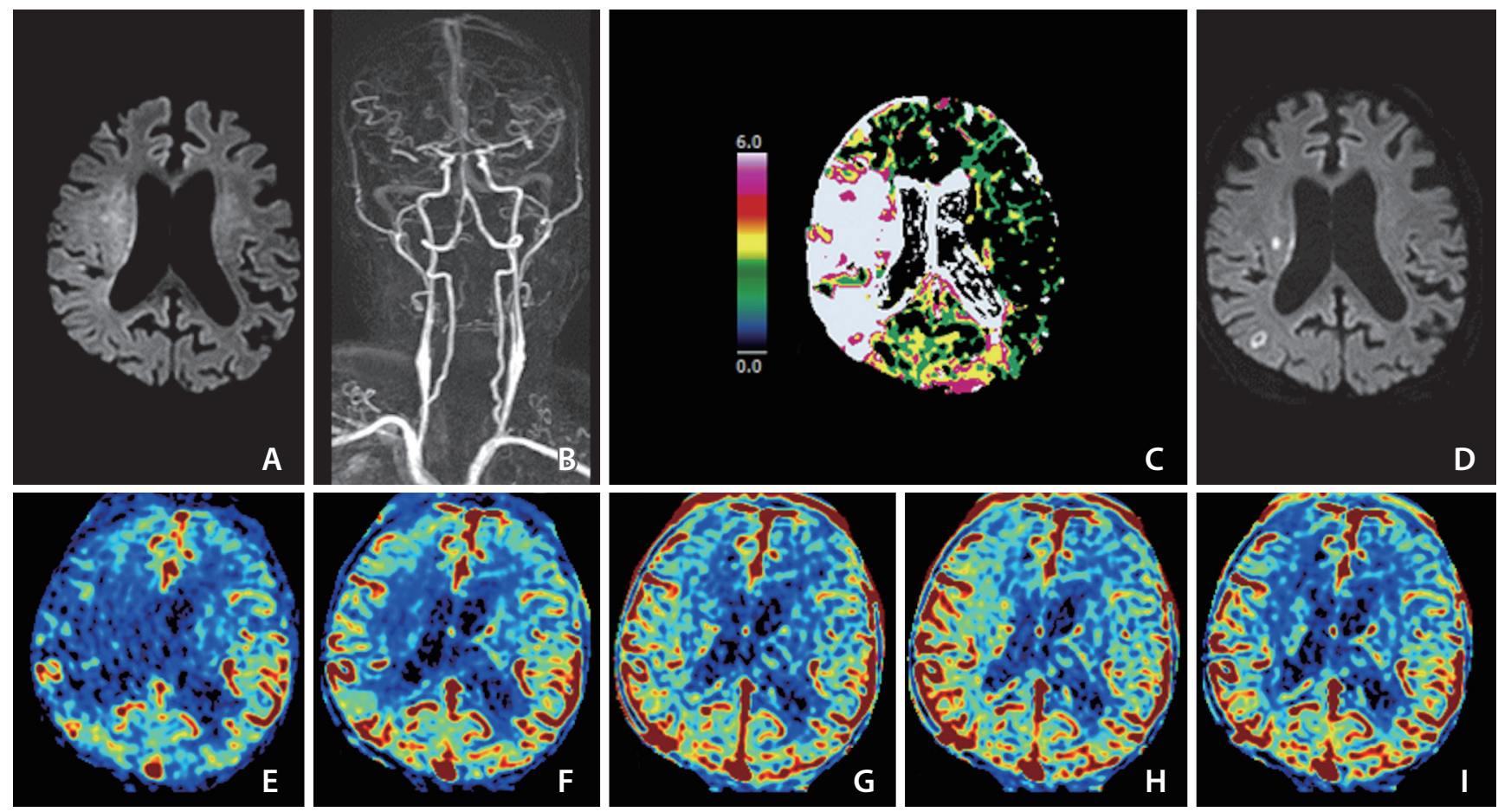

Fig. 4. Images of an elderly patient who developed left-sided weakness 4 hours prior. His premorbid modified Rankin scale score was 0 , and the National Institutes of Health Stroke Scale score at admission was 10. He was ineligible for IVT because he was taking an oral anticoagulant for arterial fibrillation. (A) Diffusion-weighted magnetic resonance imaging (DWI) at admission shows the core mainly involving more than one-third of the right middle cerebral artery (MCA) territory. (B) Dynamic contrast-enhanced magnetic resonance angiography (DCE-MRA) at admission shows partial occlusion at the first bifurcation of the right MCA. (C) MR perfusion Tmax threshold $>6$ seconds (Tmax $>6 \mathrm{~s}$ ) map at admission shows penumbra (white zone) larger than the core representing target mismatch. Catheter digital subtraction angiography performed prior to endovascular thrombectomy (EVT) revealed distal migration of fragmented thrombi to the M3 and M4 branches of the right MCA, so EVT did not undergo. (D) DWI on Day 1 shows that most of the baseline DWI lesions were reversed except for two tiny infarct signals. (E-I) Images of arterial $(\mathbf{E})$, capillary (F), early venous $(\mathbf{G})$, late venous $(\mathbf{H})$, and delay (I) phases of collateral map derived from DCE-MRA show good collateral perfusion status (MAC score of 4$)^{38}$ defined as collateral perfusion delay equal to or less than one-half of affected MCA territory in the capillary phase and no or a small delay in the early venous phase. 
favorable collateral circulation (slow progressors). ${ }^{48}$ Contrary to early window trials which had heterogeneous patients including slow and fast progressors, most patients in late window trials were slow progressors because the patients showed a small core volume even in later times. The ineffectiveness of EVT in fast progressors would neutralize the benefits of EVT in early window trials, which resulted in a better treatment effect in late window trials than in early window trials. Therefore, research integrating brain imaging (CT and DWI) and collateral perfusion imaging with consideration of the timepoint of image acquisition is necessary for the precise prediction of the ischemic core and penumbra. Advanced techniques in imaging and data science will enhance the predictability of that research.

\section{Hemorrhagic Transformation}

Symptomatic hemorrhagic transformation of acute ischemic stroke is a leading cause of unfavorable outcomes in acute ischemic stroke. Patients with acute ischemic stroke expecting hemorrhagic transformation should be excluded from revascularization treatment. Previous studies have shown that older age, higher baseline NIHSS score, high blood pressure, hyperglycemia, larger baseline DWI lesion, and poor collaterals were main predictors of symptomatic hemorrhagic transformation, ${ }^{49}$ but none of these factors has become a clinical standard due to low specificity. Permeability imaging derived from CTP, DCE-MRP, or DSC-MRP showed the possibility of an individualized prediction of hemorrhagic transformation. However, the additional long acquisition time and absence of standard application of the measurement models are limitations in the practical use of permeability imaging in acute ischemic stroke. In 2021, Lee et al. ${ }^{39}$ introduced permeability imaging derived from DCE-MRA with a 90 second acquisition time and showed a high sensitivity for the prediction of hemorrhagic transformation in patients with poor collateral perfusion status. However, further validation in a large population is necessary.

\section{Standardization of Imaging Generation and Interpretation}

Another challenge is the standardization of imaging generation and interpretation. The interrater agreement in the measurement of the NCCT ASPECTS is not satisfactory due to the inconspicuousness of early CT findings of acute ischemic stroke. In the case of perfusion imaging, image interpretation is inconsistent because of variability in the processing algo- rithm and applied thresholds. These problems may result in individual differences in the application of guidelines. Automated software for generating perfusion maps, assessment of ASPECTS, target mismatch, and large vessel occlusion has been developed. ${ }^{50}$ It provides rapid patient assessment and consistent treatment application. However, the utility of automated software can be limited if the gold standard that they use as ground truth is inaccurate (Fig. 1C). Therefore, the development of accurate imaging methods and criteria to assess the tissue viability and collateral perfusion status should take precedence. Adequate assessment and validation of algorithms used in various automated software programs are other challenging tasks.

\section{CONCLUSION}

Clinical trials have demonstrated the effectiveness of IVT and EVT in patients with acute ischemic stroke and expanded the applicable time window of those revascularization treatments. Aside from advances in treatment methods, imaging-based selection of eligible patients is crucial to success. Accordingly, the guidelines for revascularization treatments have also evolved to accept multimodal imaging evaluation. CT (NCCT, CTA [single or multiphase], and CTP) or MRI (DWI, SWI, MRA [single or multiphase], MRP, and/or FLAIR) performs well for the following imaging roles in acute ischemic stroke: 1) exclusion of intracranial hemorrhage and stroke mimic disease, 2) assessment of salvageable brain, 3) localization of the vascular occlusion and thrombus, and 4) estimation of collateral circulation. The trade-off between gaining precise information by imaging evaluation and losing time is no longer an issue because of the rapid acquisition of CT and MRI ( $<5$ minutes). However, assessing the salvageable brain by determining the core and penumbra is still not precise, and predicting hemorrhagic transformation is limited. Recently developed collateral imaging derived from dynamic imaging techniques has provided precise collateral perfusion information according to individual hemodynamics without additional time consumption. It can be expected that integration of brain imaging and precise collateral perfusion imaging with consideration of time of imaging acquisition will enable personalized assessment of the core and penumbra and prediction of hemorrhagic transformation in the near future. Sophisticated imaging methods and criteria to select eligible patients for revascularization treatments and automated 
software based on those imaging methods and criteria will open an era of precise medicine in acute ischemic stroke.

\section{Fund}

This study was supported by the National Research Foundation of Korea as subject number 'NRF-2020R1F1A1071619'.

\section{Ethics Statement}

The approval of the institutional ethics committee and informed consents were waived since its nature lies on literature review. Since the consent for publication was not available for the patients mentioned in the figures, patient's information was anonymized by removing the sex and specific age.

\section{Conflicts of Interest}

The authors have no conflicts to disclose.

\section{Author Contributions}

Concept and design: HJK and HGR. Analysis and interpretation: HJK. Data collection: HJK and HGR. Writing the article: HJK. Critical revision of the article: HJK and HGR. Final approval of the article: HJK and HGR. Statistical analysis: HJK. Obtained funding: HJK. Overall responsibility: HJK and HGR.

\section{ORCID}

Hyun Jeong Kim: https://orcid.org/0000-0002-1312-3080

Hong Gee Roh: https://orcid.org/0000-0003-0227-0971

\section{REFERENCES}

1. National Institute of Neurological Disorders and Stroke rt-PA Stroke Study Group. Tissue plasminogen activator for acute ischemic stroke. N Engl J Med 1995;333:1581-1587

2. Hacke W, Kaste M, Bluhmki E, Brozman M, Dávalos A, Guidetti D, et al.; ECASS Investigators. Thrombolysis with alteplase 3 to 4.5 hours after acute ischemic stroke. N Engl J Med 2008;359:13171329

3. Goyal M, Demchuk AM, Menon BK, Eesa M, Rempel JL, Thornton J, et al.; ESCAPE Trial Investigators. Randomized assessment of rapid endovascular treatment of ischemic stroke. $N$ Engl J Med 2015;372:1019-1030

4. Jovin TG, Chamorro A, Cobo E, de Miquel MA, Molina CA, Rovira A, et al.; REVASCAT Trial Investigators. Thrombectomy within 8 hours after symptom onset in ischemic stroke. N Engl J Med
2015;372:2296-2306

5. Saver JL, Goyal M, Bonafe A, Diener HC, Levy El, Pereira VM, et al.; SWIFT PRIME Investigators. Stent-retriever thrombectomy after intravenous t-PA vs. t-PA alone in stroke. N Engl J Med 2015;372:2285-2295

6. Berkhemer OA, Fransen PS, Beumer D, van den Berg LA, Lingsma HF, Yoo AJ, et al.; MR CLEAN Investigators. A randomized trial of intraarterial treatment for acute ischemic stroke. N Engl J Med 2015;372:11-20 Erratum in: N Engl J Med 2015;372:394

7. Campbell BC, Mitchell PJ, Kleinig TJ, Dewey HM, Churilov L, Yassi $\mathrm{N}$, et al.; EXTEND-IA Investigators. Endovascular therapy for ischemic stroke with perfusion-imaging selection. N Engl J Med 2015;372:1009-1018

8. Nogueira RG, Jadhav AP, Haussen DC, Bonafe A, Budzik RF, Bhuva P, et al.; DAWN Trial Investigators. Thrombectomy 6 to 24 hours after stroke with a mismatch between deficit and infarct. NEngl J Med 2018;378:11-21

9. Albers GW, Marks MP, Kemp S, Christensen S, Tsai JP, Ortega-Gutierrez S, et al.; DEFUSE 3 Investigators. Thrombectomy for stroke at 6 to 16 hours with selection by perfusion imaging. $N$ Engl J Med 2018;378:708-718

10. Powers WJ, Rabinstein AA, Ackerson T, Adeoye OM, Bambakidis NC, Becker K, et al. Guidelines for the early management of patients with acute ischemic stroke: 2019 update to the 2018 guidelines for the early management of acute ischemic stroke: a guideline for healthcare professionals from the American Heart Association/American Stroke Association. Stroke 2019;50:e344-e418 Erratum in: Stroke 2019;50:e440-e441

11. Liebeskind DS. Collaterals in acute stroke: beyond the clot. Neuroimaging Clin N Am 2005;15:553-573

12. Shuaib A, Butcher K, Mohammad AA, Saqqur M, Liebeskind DS. Collateral blood vessels in acute ischaemic stroke: a potential therapeutic target. Lancet Neurol 2011;10:909-921

13. Molina CA. Futile recanalization in mechanical embolectomy trials: a call to improve selection of patients for revascularization. Stroke 2010;41:842-843

14. Wufuer A, Wubuli A, Mijiti P, Zhou J, Tuerxun S, Cai J, et al. Impact of collateral circulation status on favorable outcomes in thrombolysis treatment: a systematic review and meta-analysis. Exp Ther Med 2018;15:707-718

15. Wintermark M, Rowley HA, Lev MH. Acute stroke triage to intravenous thrombolysis and other therapies with advanced CT or MR imaging: pro CT. Radiology 2009;251:619-626

16. Köhrmann M, Schellinger PD. Acute stroke triage to intravenous thrombolysis and other therapies with advanced CT or MR imaging: pro MR imaging. Radiology 2009;251:627-633 
17. Na DG, Sohn CH, Kim EY. Imaging-based management of acute ischemic stroke patients: current neuroradiological perspectives. Korean J Radio/ 2015;16:372-390

18. Schlemm L, Endres M, Werring DJ, Nolte CH. Benefit of intravenous thrombolysis in acute ischemic stroke patients with high cerebral microbleed burden. Stroke 2020;51:232-239

19. Charidimou A, Shoamanesh A; International META-MICROBLEEDS Initiative. Clinical relevance of microbleeds in acute stroke thrombolysis: comprehensive meta-analysis. Neurology 2016;87:1534-1541

20. Grotta JC, Chiu D, Lu M, Patel S, Levine SR, Tilley BC, et al. Agreement and variability in the interpretation of early $C T$ changes in stroke patients qualifying for intravenous rtPA therapy. Stroke 1999;30:1528-1533

21. Barber PA, Demchuk AM, Zhang J, Buchan AM. Validity and reliability of a quantitative computed tomography score in predicting outcome of hyperacute stroke before thrombolytic therapy. ASPECTS study group. Alberta stroke programme early CT score. Lancet 2000;355:1670-1674 Erratum in: Lancet 2000;355:2170

22. Goyal M, Menon BK, van Zwam WH, Dippel DW, Mitchell PJ, Demchuk AM, et al.; HERMES collaborators. Endovascular thrombectomy after large-vessel ischaemic stroke: a meta-analysis of individual patient data from five randomised trials. Lancet 2016;387:1723-1731

23. Purushotham A, Campbell BC, Straka M, Mlynash M, Olivot JM, Bammer R, et al. Apparent diffusion coefficient threshold for delineation of ischemic core. Int J Stroke 2015;10:348-353

24. Zaro-Weber O, Moeller-Hartmann W, Heiss WD, Sobesky J. Maps of time to maximum and time to peak for mismatch definition in clinical stroke studies validated with positron emission tomography. Stroke 2010;41:2817-2821

25. Cereda CW, Christensen S, Campbell BCV, Mishra NK, Mlynash M, Levi C, et al. A benchmarking tool to evaluate computer tomography perfusion infarct core predictions against a DWI standard. J Cereb Blood Flow Metab 2016;36:1780-1789

26. Davis SM, Donnan GA, Parsons MW, Levi C, Butcher KS, Peeters A, et al.; EPITHET investigators. Effects of alteplase beyond $3 \mathrm{~h}$ after stroke in the echoplanar imaging thrombolytic evaluation trial (EPITHET): a placebo-controlled randomised trial. Lancet Neurol 2008;7:299-309

27. Thomalla G, Boutitie F, Fiebach JB, Simonsen CZ, Nighoghossian N, Pedraza S, et al.; WAKE-UP Investigators. Stroke with unknown time of symptom onset: baseline clinical and magnetic resonance imaging data of the first thousand patients in wakeup (efficacy and safety of MRI-based thrombolysis in wake-up stroke: a randomized, doubleblind, placebo-controlled trial). Stroke 2017:48:770-773

28. Odland A, Særvoll P, Advani R, Kurz MW, Kurz KD. Are the current MRI criteria using the DWI-FLAIR mismatch concept for selection of patients with wake-up stroke to thrombolysis excluding too many patients? Scand J Trauma Resusc Emerg Med 2015;23:22

29. Broocks $G$, Leischner H, Hanning U, Flottmann F, Faizy TD, Schön $G$, et al. Lesion age imaging in acute stroke: water uptake in CT versus DWI-FLAIR mismatch. Ann Neurol 2020;88:1144-1152

30. Le Bras A, Raoult H, Ferré JC, Ronzière T, Gauvrit JY. Optimal MRI sequence for identifying occlusion location in acute stroke: which value of time-resolved contrast-enhanced MRA? AJNR Am J Neuroradiol 2015;36:1081-1088

31. Ko HC, Ryu CW, Yun SJ, Koh JS, Shin HS, Kim EJ. Characteristic signs on $\mathrm{T}^{*}$-based imaging and their relationship with results of reperfusion therapy for acute ischemic stroke: a systematic review and evidence to date. Neurointervention 2018;13:90-99

32. Menon BK, Smith EE, Modi J, Patel SK, Bhatia R, Watson TW, et al. Regional leptomeningeal score on CT angiography predicts clinical and imaging outcomes in patients with acute anterior circulation occlusions. AJNR Am J Neuroradiol 2011;32:16401645

33. Menon BK, d'Esterre CD, Qazi EM, Almekhlafi M, Hahn L, Demchuk AM, et al. Multiphase CT angiography: a new tool for the imaging triage of patients with acute ischemic stroke. Radiology 2015;275:510-520

34. Berkhemer OA, Jansen IG, Beumer D, Fransen PS, van den Berg LA, Yoo AJ, et al.; MR CLEAN Investigators. Collateral status on baseline computed tomographic angiography and intra-arterial treatment effect in patients with proximal anterior circulation stroke. Stroke 2016;47:768-776

35. Jadhav AP, Diener HC, Bonafe A, Pereira VM, Levy El, Baxter BW, et al.; SWIFT PRIME investigators. Correlation between clinical outcomes and baseline CT and CT angiographic findings in the SWIFT PRIME trial. AJNR Am J Neuroradiol 2017;38:2270-2276

36. Faizy TD, Kabiri R, Christensen S, Mlynash M, Kuraitis GM, Broocks G, et al. Favorable venous outflow profiles correlate with favorable tissue-level collaterals and clinical outcome. Stroke 2021;52:1761-1767

37. Roh HG, Kim EY, Kim IS, Lee HJ, Park JJ, Lee SB, et al. A novel collateral imaging method derived from time-resolved dynamic contrast-enhanced MR angiography in acute ischemic stroke: a pilot study. AJNR Am J Neuroradio/ 2019;40:946-953

38. Kim HJ, Lee SB, Choi JW, Jeon YS, Lee HJ, Park JJ, et al. Multiphase MR angiography collateral map: functional outcome 
after acute anterior circulation ischemic stroke. Radiology 2020;295:192-201

39. Lee TJ, Roh HG, Kim JH, Lee SB, Park JJ, Lee HJ, et al. Collateral and permeability imaging derived from dynamic contrast material-enhanced MR angiography in prediction of PH 2 hemorrhagic transformation after acute ischemic stroke: a pilot study. Neuroradiology 2021;63:1471-1479

40. Lee HJ, Roh HG, Lee SB, Jeon YS, Park JJ, Lee TJ, et al. Collateral estimation by susceptibility-weighted imaging and prediction of functional outcomes after acute anterior circulation ischemic stroke. Sci Rep 2021;11:21370

41. Lu X, Meng L, Zhou Y, Wang S, Fawaz M, Wang M, et al. Quantitative susceptibility-weighted imaging may be an accurate method for determining stroke hypoperfusion and hypoxia of penumbra. Eur Radiol 2021;31:6323-6333

42. Jones TH, Morawetz RB, Crowell RM, Marcoux FW, FitzGibbon SJ, DeGirolami U, et al. Thresholds of focal cerebral ischemia in awake monkeys. J Neurosurg 1981;54:773-782

43. Marcoux FW, Morawetz RB, Crowell RM, DeGirolami U, Halsey $J H$ Jr. Differential regional vulnerability in transient focal cerebral ischemia. Stroke 1982;13:339-346

44. Hoving JW, Marquering HA, Majoie CBLM, Yassi N, Sharma G, Liebeskind DS, et al. Volumetric and spatial accuracy of comput- ed tomography perfusion estimated ischemic core volume in patients with acute ischemic stroke. Stroke 2018;49:2368-2375

45. Zaro-Weber O, Moeller-Hartmann W, Heiss WD, Sobesky J. The performance of MRI-based cerebral blood flow measurements in acute and subacute stroke compared with 150-water positron emission tomography: identification of penumbral flow. Stroke 2009;40:2413-2421

46. Labeyrie MA, Turc G, Hess A, Hervo P, Mas JL, Meder JF, et al. Diffusion lesion reversal after thrombolysis: a MR correlate of early neurological improvement. Stroke 2012;43:2986-2991

47. Boers AMM, Jansen IGH, Beenen LFM, Devlin TG, San Roman L, $\mathrm{Heo} \mathrm{JH}$, et al. Association of follow-up infarct volume with functional outcome in acute ischemic stroke: a pooled analysis of seven randomized trials. J Neurointerv Surg 2018;10:1137-1142

48. Albers GW. Late window paradox. Stroke 2018;49:768-771

49. Boisseau W, Fahed R, Lapergue B, Desilles JP, Zuber K, Khoury N, et al.; ETIS Investigators. Predictors of parenchymal hematoma after mechanical thrombectomy: a multicenter study. Stroke 2019;50:2364-2370

50. Mokli Y, Pfaff J, Dos Santos DP, Herweh C, Nagel S. Computer-aided imaging analysis in acute ischemic stroke - background and clinical applications. Neurol Res Pract 2019;1:23 